\title{
Visible-Light Emulsion Photopolymerization of Styrene
}

\author{
Frédéric Le Quéméner, Daniel Subervie, Fabrice Morlet-Savary, Jacques Lalevée,* \\ Muriel Lansalot,* Elodie Bourgeat-Lami, * and Emmanuel Lacôte*
}

\begin{abstract}
The photopolymerization of styrene in emulsion is achieved in a conventional double-wall reactor equipped with a LED ribbon coiled around the external glass wall. Styrene mixed to acridine orange is added to the water phase containing sodium dodecyl sulfate, a water-soluble N-heterocyclic carbene-borane and disulfide, and irradiated. Highly stable latexes are obtained, with particles up to a diameter of $300 \mathrm{~nm}$. The ability to reach such large particle sizes via a photochemical process in a dispersed medium is due to the use of visible light: the photons in the visible range are less scattered by larger objects and thus penetrate and initiate better the polymerizations. They are also greener and cheaper to produce via LEDs, and much safer than UVs. The method presented does not require any specific glassware; it works at lower temperature and delivers larger particles compared to thermal processes at similar solids contents and surfactant concentrations.
\end{abstract}

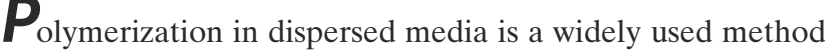
with applications in both industry and academia because it offers significant practical advantages. ${ }^{[1]}$ It generally proceeds in aqueous suspensions, that is in a cheap, safe and green solvent; it quickly leads to high molar mass polymers, and the final products are fluid latexes even for high solids contents. On the other hand, radical photopolymerizations are equally desirable, since they are triggered by light, and as such subject to external temporal and spatial control. ${ }^{[2]}$

Combining the advantages of both systems is thus of considerable interest. ${ }^{[3]}$ However, photopolymerizations

[**] F. Le Quéméner, D. Subervie, Dr. M. Lansalot, Dr. E. Bourgeat-Lami, Dr. E. Lacôte

Univ Lyon, Université Claude Bernard Lyon 1, CPE Lyon, CNRS, C2P2

43 Bd du 11 novembre 1918, 69616 Villeurbanne (France)

E-mail: muriel.lansalot@univ-lyon1.fr

elodie.bourgeat-lami@univ-lyon1.fr

emmanuel.lacote@univ-lyon1.fr

D. Subervie, Dr. E. Lacôte

Univ Lyon, Université Claude Bernard Lyon 1, CNRS, CNES,

ArianeGroup, LHCEP, Bât. Raulin

2 rue Victor Grignard, 69622 Villeurbanne (France)

Dr. F. Morlet-Savary, Prof. J. Lalevée

Institut de Science des Matériaux de Mulhouse (IS2M)-

UMR CNRS 7361-UHA

15 rue Jean Starcky, 68057 Mulhouse (France)

E-mail: jacques.lalevee@uha.fr require photoinitiating systems (PIS) that are almost always lipophilic and sensitive to UV light. Initiation thus takes place in the organic phase. Hence UV photopolymerization in microemulsions ${ }^{[4]}$ or miniemulsions ${ }^{[5]}$ are better suited, since they favor the localization of the PIS in the small dispersed organic droplets. These are important results, yet they suffer from some drawbacks. Microemulsions require a large amount of surfactant. ${ }^{[6]}$ Miniemulsions are better from that point of view; however, they require highly energetic homogeneization devices. ${ }^{[7]}$

Emulsion photopolymerizations have been attempted as well. However, they differ from the previous methods in that their initiation early stages should take place in the water phase. Previous efforts have examined various systems to induce initiation in the water phase,$^{[8]}$ but also in the micellar phase, and then very specific methods (use of magnetic fields) have to be found to ensure efficiency. ${ }^{[9]}$ Inverse emulsion photopolymerization was also studied, ${ }^{[10]}$ although its mechanism should be better described as microsuspension polymerization.

All of these techniques however suffer from drawbacks: low conversions, low solids contents, the need for specific equipment, and/or poor latex stability. Furthermore, they are usually restricted to the formation of small particles (typically $20-140 \mathrm{~nm}$ ). This is a severe limitation if one wants to access larger objects, and it stems from the use of UV light from mainly $\mathrm{Hg}$ lamps to trigger the radical reactions. UV rays have severe drawbacks. They are highly energetic and harmful to the operators, necessitating special protection measures; furthermore, they do not penetrate thick samples well because they are scattered by the large monomer droplets and/or polymer particles. The penetration issue can be offset by running the polymerizations in a tubular vessel, but the diameter of the particles remains below $100 \mathrm{~nm} .^{[11]}$

We felt that we could access both small and large particles by using visible light, which is cheap and greener to produce via LEDs. These photons are also safer (less energetic), thus again cheaper. Because their wavelengths are longer, they are much less scattered by larger objects and thus penetrate better. And from a technological point of view, the LEDs are very compact, long-lived and do not emit heat or ozone. They can therefore be easily included into chemical reactors. As a consequence, visible-light mediated radical reactions have grown exponentially over the last few years in both molecular $^{[12]}$ and polymer chemistry. ${ }^{[13]}$

Oddly though, despite an early isolated report, ${ }^{[14]}$ only very few works focus on visible-light photopolymerizations in dispersed media, ${ }^{[15]}$ and almost none involving conventional 
emulsion polymerization. ${ }^{[15 a-c]}$ In particular, Tauer and coworkers reported that a bis(acyl)germane or phosphine oxide was cleaved under visible light and initiated photopolymerization. ${ }^{[15 b]}$ However, because Type I photoinitiators are lipophilic, very large amounts of surfactant (thirteen times the critical micelle concentration, $\mathrm{CMC}$ ) were required, in conditions close to those used in microemulsion. This allowed an acceptable solids content $(20 \%)$, but the process also generated coagulum (as did the initial report ${ }^{[14]}$ ). Very recently, Li and co-workers ${ }^{[15 c]}$ showed that radical polymerization of styrene could be initiated by a visible-light photooxidizing dye, which generated radicals upon reaction with a specific $\mathrm{N}$-oxide surfactant. The initiation thus presumably happened at the monomer droplet surface, but there was no indication that stable latexes were formed by this method.

Some of us have introduced N-heterocyclic carbeneboranes (NHC-boranes) as photoinitiating species for radical photopolymerizations. ${ }^{[16]}$ In particular we found mild conditions for visible-light photopolymerization in organic solution. ${ }^{[17]}$ This latter system relies on the photoreduction of diphenyldisulfide by visible-light irradiation of acridine orange (AO). This generates a thiyl radical (see Scheme 1, step 1), which further abstracts a hydrogen atom from the NHC-borane, leading to the initiating NHC-boryl radical (step 2).

Given the dearth of efficient systems to initiate emulsion photopolymerizations, we considered NHC-boranes as potential solutions to this challenge. According to the previously mentioned accepted mechanism of emulsion polymerization, initiation must proceed in the aqueous phase. After the addition of a few monomer units (2 and 3 in the case of styrene under thermal potassium persulfate (KPS) initiation, ${ }^{[18]}$ see Scheme 1, steps 3 and 4), the small oligomers become insoluble and the polymerization then proceeds to form particles stabilized by the surfactant (step 5). Thus we needed to translate our previous organosoluble initiation to the water phase, and therefore design a fully water-soluble borane-based initiating system that should still work adequately in water (Figure 1). Radical

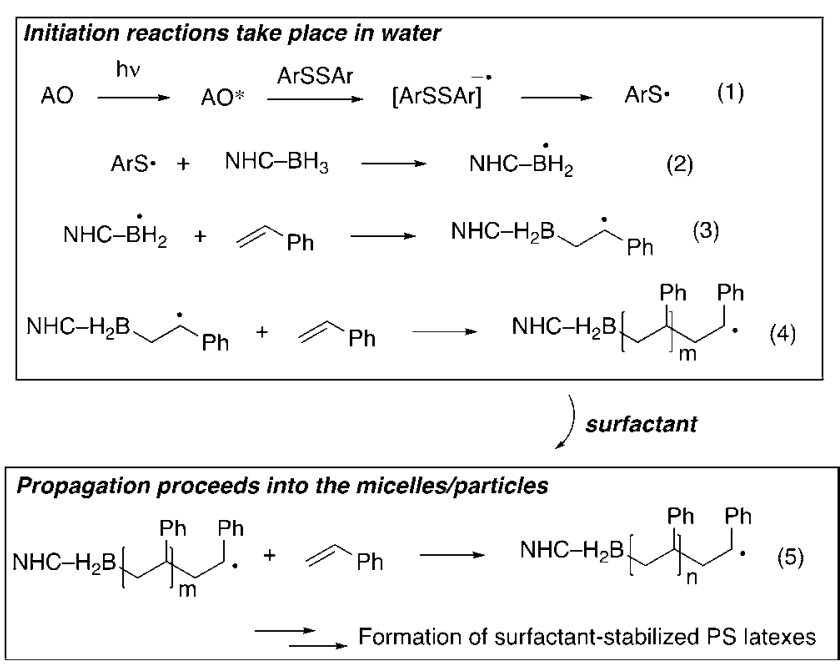

Scheme 1. Proposed mechanism for the emulsion photopolymerization when the surfactant concentration is above its CMC.

$$
\stackrel{\mathrm{N}_{+}}{\stackrel{\mathrm{N}}{\mathrm{N}}>-}-\mathrm{BH}_{3}
$$

1 (hydrosoluble)

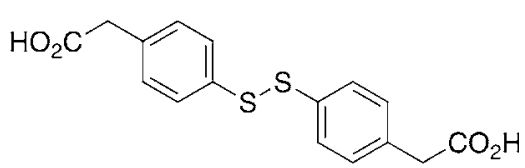

2 (hydrosoluble)

Figure 1. Structures of the compounds used in this work.

reactions are generally compatible with water, but can nonetheless be strongly affected, especially if water is able to develop hydrogen bonds with the reacting radicals. ${ }^{[19]}$

We selected the water-soluble triazolylidene-borane $\mathbf{1}$ as the boron reagent, ${ }^{[20]}$ and disulfide $\mathbf{2}$ as the disulfide partner. $\mathbf{2}$ is negatively charged above its $\mathrm{pKa}$ (3.8) and thus highly water-soluble. Partitioning experiments were carried out with AO, which showed that while it is soluble in both phases, AO prefers the aqueous phase $\left([\mathbf{A O}]_{\mathrm{aq}} /[\mathbf{A O}]_{\mathrm{St}}=167\right)$. Thus we kept this dye for this work.

In a typical polymerization procedure (see Table 1, entry 1), a solution of the dye and styrene was added to the water phase containing $\mathbf{1}, \mathbf{2}$, and the surfactant (sodium dodecyl sulfate, SDS, at twice the CMC). We used a conventional double-wall reactor equipped with a LED ribbon coiled around the external glass walls (see the Supporting Information for details). AO was initially solubilized in the monomer to prevent any undesired initiation of the polymerization that might arise from its premature reaction with the disulfide.

Gratifyingly, the conversion was total after $6 \mathrm{~h}$ of irradiation (Figure 2), and the latex obtained proved highly stable, without the presence of any coagulum. This is an important result for NHC-borane-initiated photopolymerization, which thus far was limited to the polymerization of acrylates in homogeneous media. Furthermore, the average particle size measured by dynamic light scattering (DLS) was $112 \mathrm{~nm}$, with a polydispersity index (PdI) of 0.02. However, TEM analysis showed that the particle size distribution (PSD) was rather large $\left(D_{\mathrm{w}} / D_{\mathrm{n}}=1.3\right)$ with a significantly smaller numberaverage diameter $\left(D_{\mathrm{n}}\right)$ of $67 \mathrm{~nm}$. The difference in average size determined by the two methods stems from the fact that DLS emphasizes larger objects at the expense of smaller ones. While increasing the concentration of AO did not affect the polymerization, doubling the relative amounts of both $\mathbf{1}$ and $\mathbf{2}$ resulted in a better PSD $\left(D_{\mathrm{w}} / D_{\mathrm{n}}=1.1\right)$ and an increased average size (91 nm, entry 2).

We next carried out a series of control experiments (Figure 2, right hand side). Without the dye, the polymerization was less efficient (plateau at $80 \%$ conversion after $6 \mathrm{~h}$, entry 3 ). It should be noted that the thiyl radicals are formed by irradiation far from the absorption maximum of the disulfide. ${ }^{[21]}$ Removing the NHC-borane from the photoinitiating system (but leaving AO) suppressed the polymerization (entry 4). Similarly no conversion was observed in the absence of disulfide (entry 5), when the polymerization 
Table 1: Visible-light emulsion photopolymerization of styrene.

$$
\begin{aligned}
& \left.1 \text { (1 equiv.; [1] }=0.11 \mathrm{gL}^{-1}\right) \\
& 2(0.5 \text { equiv. }) . \mathrm{AO}(0.01 \text { equiv.) } \\
& \frac{\mathrm{Na}_{2} \mathrm{CO}_{3}}{\mathrm{H}_{2} \mathrm{O}, \mathrm{rt}} \\
& \text { visible light (LED) }
\end{aligned}
$$

\begin{tabular}{|c|c|c|c|c|c|c|c|}
\hline Entry $^{[a]}$ & $\begin{array}{l}c(\mathrm{SDS}) \\
(x \mathrm{CMC})\end{array}$ & $\begin{array}{l}\text { Conv. } \\
{[\%]}\end{array}$ & $\begin{array}{l}\text { Time } \\
{[\mathrm{h}]}\end{array}$ & $\begin{array}{l}D_{\mathrm{h}} \\
{[\mathrm{nm}]^{[\mathrm{b}]}}\end{array}$ & $\mathrm{PDI}^{[\mathrm{b}]}$ & $\begin{array}{l}D_{\mathrm{n}} \\
{[\mathrm{nm}]^{[\mathrm{c}]}}\end{array}$ & $\begin{array}{l}D_{w} / \\
D_{n}[c]\end{array}$ \\
\hline 1 & 2 & 100 & 6 & 112 & 0.02 & 67 & 1.3 \\
\hline $2^{[\mathrm{d}]}$ & 2 & 96 & 6 & 110 & 0.04 & 91 & 1.1 \\
\hline $3^{[\mathrm{e}]}$ & 2 & 80 & 6 & 140 & 0.05 & - & - \\
\hline $4^{[f]}$ & 2 & 0 & 6 & - & - & - & - \\
\hline $5^{[g]}$ & 2 & 0 & 6 & - & - & - & - \\
\hline $6^{[\mathrm{h}]}$ & 2 & 0 & 6 & - & - & - & - \\
\hline $7^{[]]}$ & 2 & 0 & 6 & - & - & - & - \\
\hline $8^{[j]}$ & 2 & 42 & 6.5 & 93 & 0.05 & - & - \\
\hline $9^{[j]}$ & 16.5 & 97 & 4.5 & 66 & 0.11 & - & - \\
\hline 10 & 0.5 & 86 & 50 & 330 & 0.03 & 294 & 1.01 \\
\hline 11 & 0.75 & 100 & 16 & 330 & 0.03 & 153 & 1.03 \\
\hline 12 & 1 & 92 & 9 & 183 & 0.02 & 94 & 1.32 \\
\hline 13 & 1.5 & 100 & 8 & 142 & 0.03 & 80 & 1.24 \\
\hline $14^{[\mathrm{k}]}$ & 2 & 97 & 4 & 97 & 0.03 & 46 & 1.52 \\
\hline $15^{[l]}$ & 2 & 100 & 10 & 136 & 0.01 & 81 & 1.37 \\
\hline $16^{[\mathrm{m}]}$ & 2 & 100 & 11 & 153 & 0.02 & 68 & 1.71 \\
\hline $17^{[n]}$ & 0.75 & 100 & 3.5 & 93 & 0.02 & 79 & 1.06 \\
\hline $18^{[\mathrm{n}]}$ & 1 & 100 & 0.8 & 88 & 0.05 & 69 & 1.06 \\
\hline $19^{[n]}$ & 2 & 100 & 0.6 & 68 & 0.02 & 52 & 1.06 \\
\hline
\end{tabular}

[a] Conditions: a solution of $\mathrm{AO}$ ( $1 \mathrm{~mol} \%$ with regard to 1 ) and styrene was added to the water phase containing the surfactant (SDS),

$1\left([1]=0.11 \mathrm{~g} \mathrm{~L}^{-1}\right)$ and 2 (50 mol\% with regard to 1). Unless otherwise specified, the solids content was fixed at $20 \%$. [b] Hydrodynamic diameter and polydispersity index determined by DLS. [c] Numberaverage diameter and size dispersity determined by TEM. [d] Polymerization carried out with twice the concentration of 1 and 2, [AO] unchanged. [e] Polymerization carried out without AO. [f] Polymerization carried out without NHC-borane. [g] Polymerization carried out without disulfide. [h] Polymerization carried out in the dark. [i] Polymerization carried only with disulfide under irradiation (and the surfactant). [j] The organosoluble photoinitiating system PhSSPh/3 was used. [k] Solids content was $13 \%$. [l] Solids content was $26 \%$. [m] Solids content was $31 \%$. [n] Standard thermal initiation was used (KPS, $4.6 \times 10^{-3} \mathrm{~mol} \mathrm{~L}^{-1}$ ).
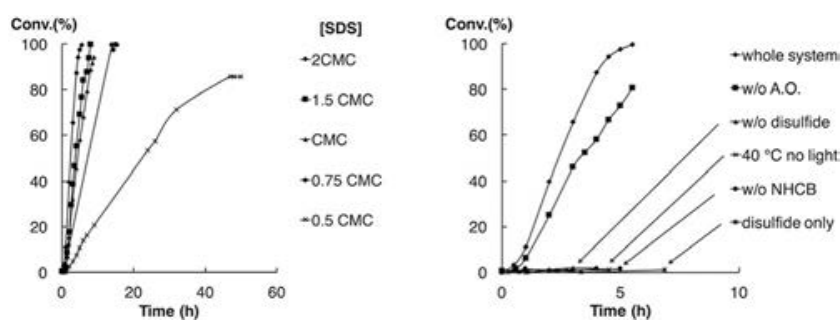

Figure 2. Conversion profiles of the photopolymerizations for different surfactant concentrations (left; see Table 1, entries 1 and 10-13 for conditions) and during control experiments (right; see Table 1, entries 1 and 3-7 for conditions).

medium was left in the dark (entry 6), or when only the disulfide was present (entry 7). When the lipophilic NHCborane $\mathbf{3}$ was used instead of $\mathbf{1}$ in the presence of diphenyldisulfide ( $\mathrm{PhS}-\mathrm{SPh}$ ), the conversion dropped to $42 \%$ (entry 8 ). With a very large excess of surfactant $(16.5 \mathrm{CMC})$, the photopolymerization using $\mathbf{3}$ and $\mathrm{PhS}-\mathrm{SPh}$ was very efficient (97\% conversion after $4.5 \mathrm{~h}$, entry 9 ).
Overall, the previous controls show that the NHC-boryl radicals are essential for the polymerization to proceed, as nothing happens without the presence of $\mathbf{1}$ or $\mathbf{2}$ (compare entries 1 to 4 and 7). The dye is needed to get complete conversion, and cannot initiate the polymerization by itself (entry 5). Nonetheless, some radicals are still formed by irradiation of the disulfides (compare entries 1 and 3), whereby the $\mathrm{S}-\mathrm{S}$ bond of the disulfide is directly homolyzed in a Type I mechanism (ArS-SAr $\rightarrow 2 \mathrm{ArS})^{\circ}$ ). However the NHC-borane is required for the polymerization to proceed (compare entries 3 and 7).

In support of these observations, we determined the rate constants for the elementary steps involved in the polymerization initiation. The values we reported in the past were all measured in an organic solvent (and for acrylate monomers) using laser flash photolysis (LFP) ${ }^{[16 c, d]}$ By applying the same techniques to $\mathbf{1}$ and $\mathbf{2}$ in water, we could determine that the $\mathrm{H}$ atom transfer from $\mathbf{1}$ to the ArS radical derived from $\mathbf{2}$ proceeded with a rate $k_{\mathrm{H}}=5 \times 10^{7} \mathrm{~L} \mathrm{~mol}^{-1} \mathrm{~s}^{-1}$. The latter added to styrene too slowly to be recorded by LFP. On the contrary, NHC-boryl radical $\mathbf{1}^{\bullet}$ adds to styrene at a rapid rate $\left(k_{\text {add }}=5 \times 10^{6} \mathrm{~L} \mathrm{~mol}^{-1} \mathrm{~s}^{-1}\right)$, making the full system a good photoinitiating system. We have shown that NHC-boranes are outstanding additives that significantly increase the speed of bulk photopolymerizations of acrylates initiated by several Type I photoinitiators (including diphenyl disulfide). ${ }^{[22]}$ We see here that this feature is maintained in the emulsion photopolymerization of styrene. The main difference is that the flux of thiyl radicals directly generated in the continuous phase $^{[21]}$ is not enough to support a photopolymerization in the dispersed medium in the absence of the boron reagent.

The fact that the hydrophobic $\mathbf{3}$ is much less efficient than 1 to trigger the emulsion photopolymerization (compare entries 1 and 8 ) is compatible with the general mechanism envisaged (there is much less NHC-borane in the water phase). The conditions described in Entry 9 (in the presence of a huge amount of surfactant) are close to that of microemulsion, which is characterized by the fact that the initial micellar medium is thermodynamically stable. Consequently, the polymerization proceeds in the micelles, where $\mathbf{3}$ is soluble, and the particles obtained should be smaller. That is what we observed in entry 9 (average diameter of $66 \mathrm{~nm}$ by DLS vs. $112 \mathrm{~nm}$ under the conditions of entry 1). We did however not attempt to develop further this aspect as it requires a too large amount of surfactant.

With the emulsion photopolymerization conditions in hands, we determined the influence of the reaction parameters. A gradual lowering of the surfactant concentration led to a growth of $D_{\mathrm{n}}$ from $67 \mathrm{~nm}$ at $2 \mathrm{CMC}$ to $294 \mathrm{~nm}$ at $0.5 \mathrm{CMC}$ (entries 10-13), albeit the polymerizations took gradually longer (from $6 \mathrm{~h}$ to $50 \mathrm{~h}$ to reach full conversion, Figure 2 left hand side). Interestingly, below the CMC of SDS, the size dispersity $\left(D_{\mathrm{w}} / D_{\mathrm{n}}\right)$ dramatically improved, from 1.32 at $1 \mathrm{CMC}$ (entry 12) to 1.03 and 1.01 at 0.75 and $0.5 \mathrm{CMC}$, respectively (entries 10 and 11). This likely reflects the time the photopolymerization takes to reach steady state. The photochemical system generates initiating radicals rather slowly, leading to a low nucleation rate. Therefore nucleation in the micelles above $\mathrm{CMC}$ becomes an issue, leading to 
higher dispersities in that case. In the presence of more NHCborane and disulfide (entry 2), more radicals are formed, and the steady state is reached faster, leading to an improved dispersity. The particles size growth suggests that coagulation events play a significant role in the polymerization. We think this is because the initiating NHC-boryl radicals are not charged (as opposed to those derived from KPS).

The result described in entry 10 is of particular importance, since the particles formed are the largest reported to date by photopolymerization in dispersed media. Their diameter is indeed high enough to significantly scatter UV light, and the better light penetration of visible light gives this technique a huge advantage compared to UV-initiated emulsion photopolymerization.

We next changed the solids content of the photopolymerization at a surfactant concentration of 2 CMC (entries 14 16). The photopolymerization was faster at the lower solids content of $13 \%$ ( $4 \mathrm{~h}$ to reach completion, entry 14 ). Conversely at the higher solids contents (26\% and $31 \%)$, the system needed more time to reach completion (10-11 h, entries 15 and 16), but the polymerizations remained efficient even if the turbidity was significantly higher. The particle sizes distribution was also noticeably worse at higher solids contents. Like the SDS content, the monomer amount likely affects the localization of the different species and thus the polymerization mechanism.

Finally, to obtain a comparison with the classical thermal conditions, we carried out three KPS-initiated emulsion polymerizations of styrene (at $0.75,1$, and $2 \mathrm{CMC}$, entries 17-19). The thermal polymerizations were faster, reaching completion 4 to 10 times faster than the photochemical polymerizations. Also the PSD was narrower above $\mathrm{CMC}$, and similar throughout all the trials (compare for example Figure 3 top and bottom right distributions, obtained thermally, to the bottom left distribution, obtained photochemically). However, the sizes obtained under the photochemical conditions were always larger. This effect was small at CMC or above CMC ( $67 \mathrm{~nm}$ vs. $52 \mathrm{~nm}$ at $2 \mathrm{CMC}, 94 \mathrm{~nm}$ vs. 69 at CMC), but it became especially strong below CMC (153 vs. $79 \mathrm{~nm}$ at $0.75 \mathrm{CMC}$ ).

To conclude, we showed that styrene can be photopolymerized in emulsion with very high conversions, and no formation of coagulum. Our method is operationally simple, green, and cheap. It delivers particles of all sizes with diameters from $46 \mathrm{~nm}$ up to $300 \mathrm{~nm}$. The key to obtain the large objects in dispersed media under photopolymerization conditions is to use visible light. Our method also works at lower temperature than the traditional thermal emulsion polymerizations and delivers larger particles and higher molar masses at the same surfactant concentration. We evidenced a critical influence of the latter also on the particle size distributions. While below the CMC the polydispersities remain low, they are higher above it. Both features are a consequence of the lower flux of initiating radicals under the photochemical conditions. Using light to trigger the polymerization also enables temporal control of the process (on/off polymerization). Further work will focus on learning more about the mechanism, and polymerizing other monomers and co-monomers. Beyond, we wish to examine how the precise spatial control that photoinitiation allows can affect the polymerization outcomes.

\section{Acknowledgements}

We thank Université Claude Bernard Lyon 1, CNRS, CPE Lyon, UHA and ANR (grants NHCX and Photo-B) for funding of this work. D.S. acknowledges Université Claude Bernard for a graduate fellowship.

\section{Conflict of interest}

The authors declare no conflict of interest.

Keywords: boron · emulsions · photochemistry . polymerization radical reactions

[1] Emulsion Polymerization and Emulsion Polymers (Eds.: P. A. Lovell, M. S. El-Aasser), Wiley, Chichester, 1997.

[2] a) J. P. Fouassier, J. Lalevée in Photoinitiators for Polymer Synthesis, Wiley-VCH, Weinheim, 2012; b) J. Lalevée, J.-P. 
Fouassier in Dyes and Chromophores in Polymer Science, Wiley, Hoboken, 2015.

[3] For two pioneering studies, see: a) M. Takeishi, H. Yoshida, S Niino, S. Hayama, Makromol. Chem. 1978, 179, 1387-1391; b) J.-P. Fouassier, D.-J. Lougnot, J. Appl. Polym. Sci. 1987, 34, $477-488$.

[4] a) C. Schauber, G. Riess, Makromol. Chem. 1989, 190, 725-735; b) M. T. Carver, U. Dreyer, R. Knoesel, F. Candau, R. M. Fitch, J. Polym. Sci. Part A 1989, 27, 2161-2177; c) G. David, F. Özer, B. C. Simionescu, H. Zareie, E. Pişkin, Eur. Polym. J. 2002, 38, 73-78; d) K. Jain, J. Klier, A. B. Scranton, Polymer 2005, 46, $11273-11278$.

[5] a) A. Chemtob, B. Kunstler, C. Croutxé-Barghorn, S. Fouchard, Colloid Polym. Sci. 2010, 288, 579-587; b) F. Jasinski, E. Lobry, A. Chemtob, C. Croutxé-Barghorn, A. Criqui, Macromol. Chem. Phys. 2013, 214, 1669-1676; c) E. Lobry, F. Jasinski, M. Penconi, A. Chemtob, C. Croutxé-Barghorn, E. Oliveros, A. M. Braun, A Criqui, RSC Adv. 2014, 4, 43756-43759.

[6] "Microemulsion Polymerization": C.-S. Chern in Encyclopedia of Polymer Science and Technology, Wiley, Hoboken, 2002.

[7] J. M. Asua, Prog. Polym. Sci. 2014, 39, 1797-1826.

[8] a) J. Kwak, P. Lacroix-Desmazes, J. J. Robin, B. Boutevin, N. Torres, Polymer 2003, 44, 5119-5130; b) S. Mah, D. Koo, H. Jeon, S. Kwon, J. Appl. Polym. Sci. 2002, 84, 2425-2431; c) M. V. Encinas, A. M. Rufs, S. G. Bertolotti, C. M. Previtali, Polymer 2009, 50, 2762-2767; d) X. Hu, J. Zhang, W. Yang, Polymer 2009, 50, $141-147$; e) T. Yamamoto, S. Seki, R. Fukae, O. Sangen, M. Kamachi, Polym. J. 1990, 22, 567-571.

[9] N. J. Turro, M.-F. Chow, C.-J. Chung, C.-H. Tung, J. Am. Chem. Soc. 1980, 102, $7391-7393$.

[10] a) S. K. Ghosh, B. M. Mandal, Polymer 1993, 34, 4287-4290; b) L. Liu, W. Yang, J. Polym. Sci. Part A 2004, 42, 846-852.

[11] P. Laurino, H. F. Hernandez, J. Bräuer, K. Krüger, H. Grützmacher, K. Tauer, P. H. Seeberger, Macromol. Rapid Commun. 2012, 33, 1770-1774.

[12] a) B. König, Eur. J. Org. Chem. 2017, 1979-1981; b) J. K. Matsui, S. B. Lang, D. R. Heitz, G. A. Molander, ACS Catal. 2017, 7 , $2563-2575$; c) M. D. Kärkäs, J. A. Porco, C. R. J. Stephenson, Chem. Rev. 2016, 116, $9683-9747$.

[13] N. Zivic, M. Bouzrati-Zerelli, A. Kermagoret, F. Dumur, J.-P. Fouassier, D. Gigmes, J. Lalevée, Chem CatChem 2016, 8, 1617 1631.

[14] I. Hudec, D. Mikulasova, P. Citovicky, V. Chrastova, Chem. Zvesti 1983, 37, 485-494.

[15] a) M. Ratanajanchai, D. Tanwilai, P. Sunintaboon, J. Colloid Interface Sci. 2013, 409, 25 - 31; b) K. Krüger, K. Tauer, Y. Yagci,
N. Moszner, Macromolecules 2011, 44, 9539-9549; c) K. Shen, Y. Jiang, Z. Liu, D. Qi, H. Wang, Y. Li, Macromol. Chem. Phys. 2015, 216, 1990-1996; d) J. Yeow, J. Xu, C. Boyer, ACS Macro Lett. 2015, 4, 984-990; e) J. Tan, H. Sun, M. Yu, B. S. Sumerlin, L. Zhang, ACS Macro Lett. 2015, 4,1249-1253;f) K. Jung, J. Xu, P. B. Zetterlund, C. Boyer, ACS Macro Lett. 2015, 4, 1139-1143.

[16] a) S. Telitel, S. Schweizer, F. Morlet-Savary, B. Graff, T. Tschamber, N. Blanchard, J. P. Fouassier, M. Lelli, E. Lacôte, J. Lalevée, Macromolecules 2013, 46, 43-48; b) M.-A. Tehfe, J. Monot, M. Malacria, L. Fensterbank, J.-P. Fouassier, D. P. Curran, E. Lacôte, J. Lalevée, ACS Macro Lett. 2012, 1, $92-$ 95; c) M.-A. Tehfe, J. Monot, M. M. Brahmi, H. Bonin-Dubarle, D. P. Curran, M. Malacria, L. Fensterbank, E. Lacote, J. Lalevée, J.-P. Fouassier, Polym. Chem. 2011, 2, 625-631; d) M.-A. Tehfe, M. Makhlouf Brahmi, J.-P. Fouassier, D. P. Curran, M. Malacria, L. Fensterbank, E. Lacôte, J. Lalevée, Macromolecules 2010, 43, $2261-2267$

[17] a) P. Xiao, J. Zhang, F. Dumur, M. A. Tehfe, F. Morlet-Savary, B. Graff, D. Gigmes, J. P. Fouassier, J. Lalevée, Prog. Polym. Sci. 2015, 41, 32-66; b) C. Dietlin, S. Schweizer, P. Xiao, J. Zhang, F. Morlet-Savary, B. Graff, J.-P. Fouassier, J. Lalevée, Polym. Chem. 2015, 6, 3895-3912.

[18] S. C. Thickett, R. G. Gilbert, Polymer 2007, 48, 6965-6991.

[19] a) G. Litwinienko, A. L. J. Beckwith, K. U. Ingold, Chem. Soc. Rev. 2011, 40, 2157-2163; b) R. E. Galian, G. Litwinienko, J. Pérez-Prieto, K. U. Ingold, J. Am. Chem. Soc. 2007, 129, 92809281; c) G. Litwinienko, K. U. Ingold, Acc. Chem. Res. 2007, 40, $222-230$

[20] S.-H. Ueng, L. Fensterbank, E. Lacôte, M. Malacria, D. P. Curran, Org. Lett. 2010, 12, 3002-3005.

[21] A similar observation has been made by the Gescheidt/BarnerKowollik groups on another (UV) photoinitiating system that works better far from its absorption maximum, although no explanation has been found yet for this behavior. See: D. E. Fast, A. Lauer, J. P. Menzel, A.-M. Kelterer, G. Gescheidt, C. BarnerKowollik, Macromolecules 2017, 50, 1815-1823.

[22] J. Lalevée, S. Telitel, M. A. Tehfe, J. P. Fouassier, D. P. Curran, E. Lacôte, Angew. Chem. Int. Ed. 2012, 51, 5958-5961; Angew. Chem. 2012, 124, 6060-6063. 